\title{
Subclustering in Cooling and Non-cooling Flow Clusters
}

\author{
P. Flin ${ }^{1,2}$, J. Krywult ${ }^{1}$ \\ ${ }^{1}$ Pedagogical University, Institute of Physics, ul. Swietokrzyska 15, \\ 25-406 Kielce, Poland \\ ${ }^{2}$ Joint Institute for Nuclear Research, Bogoliubov Laboratory of \\ Theoretical Physics, Dubna, Moscow Region 141 980, Russia
}

\begin{abstract}
For 20 hot luminous Beppo-SAX galaxy clusters the occurrence of substrucures has been investigated in 2-D optical data, mainly through the application of the wavelet analysis. Our results are compared with others, coming from 3-D optical data, as well as X-rays. In this sample of galaxy clusters a higher percentage of substructure is observed among non-cooling flow clusters than cooling flow clusters.
\end{abstract}

The properties of galaxies in clusters depend on their environment. In clusters, morphology segregation is found among galaxies both in substructures and outside them (Plionis 2001, Biviano et al. 2002). Moreover, dynamically young clusters are much more clustered spatially and cluster ellipticity is correlated with rerdshift (Plionis 2001, 2002).

In our analysis, we considered 20 Beppo-SAX clusters, for which the information on the existence, or not, of cooling flow is known (De Grandi \& Molendi 2001, 2002). All optical data, used by us, come from scanned photographic plates taken with 48-inch Schmidt Telescopes. The X-ray subclustering was studied mainly by Jones \& Forman (1999) and Schuecker et al. (2001).

Detection of structures in the optical regions under investigation was achieved using the wavelet analysis (Escalera et al. 1994) with a radial function called the Mexican Hat. For the analysis presented here, the discrete wavelet was computed on a grid of pixels for seven scales increasing from $a=8$ to 64 (in pixel units). In order to avoid any edge effects, areas larger than the cluster itself were analyzed. For each cluster and each scale $a$, the wavelet analysis was carried out on a set of 1000 randomly generated distributions of galaxies containing the same number of points as in the actual fields. It has been assumed that a substructure is real if the probability that the detected substructure having at least 4 galaxies is due to random fluctuations is less than $1 \%$.

The first column of Table 1 contains the cluster name, the second one the existence of the cooling flow or its absence, denoted as CF and NCF respectively. Column 3 gives cluster morphology ( $\mathrm{U}$ - unimodal, $\mathrm{S}$ - substructures present in the cluster), as determined by the present authors from 2-D data and wavelet analysis, column 4, some others determinations based on optical 3-D data. The last three columns give the existence of subclustering in X-ray studies, namely Jones \& Forman (1999) classification (Einstein data), Schuecker et al. (2001) investigations (based on ROSAT data), and some other studies of individual clusters also taken from the literature. The clusters without sub- 
Table 1. Properties of investigated clusters

\begin{tabular}{|c|c|c|c|c|c|c|c|c|c|c|c|c|c|}
\hline Cluster & Morph. & & Subst & $\operatorname{cts}$ & & & Cluster & Morph. & & Subs & ruct & & \\
\hline A85 & $\mathrm{CF}$ & $\mathrm{U}$ & $\mathrm{S}, \mathrm{U} ?$ & $\mathrm{P}$ & S? & & A2142 & $\mathrm{CF}$ & $\mathrm{U}$ & & $\mathrm{U}$ & $\mathrm{S}$ & \\
\hline A119 & $\mathrm{NCF}$ & $\mathrm{U}$ & $\mathrm{U}$ & $\mathrm{P}$ & $\dot{S}$ & & A2199 & $\mathrm{CF}$ & $\mathrm{s}$ & $\mathrm{S}$ & $\mathrm{E}$ & & \\
\hline $\mathrm{A} 426$ & $\mathrm{CF}$ & $\mathrm{S}$ & $\mathrm{U} ?$ & $\mathrm{E}$ & $\tilde{U}$ & & A2256 & NCF & $\mathrm{U}$ & $\mathrm{E}$ & $\mathrm{S}$ & $\mathrm{S}$ & \\
\hline A 496 & $\mathrm{CF}$ & $\mathrm{S}$ & & $\bar{U}$ & & $\mathrm{~S}$ & A2319 & NCF & & & $\mathrm{O}$ & & $\mathrm{S}$ \\
\hline A754 & $\mathrm{NCF}$ & $\mathrm{S}$ & $\mathrm{S}$ & $\mathrm{E}$ & $\mathrm{S}$ & & A3266 & NCF & $\mathrm{U}$ & $\mathrm{U}$ & $\mathrm{E}$ & $\mathrm{S}$ & \\
\hline A1367 & $\mathrm{NCF}$ & $\mathrm{S}$ & $\mathrm{U}$ & $\mathrm{E}$ & $\mathrm{S}$ & & A3376 & $\mathrm{NCF}$ & $\mathrm{S}$ & $\mathrm{U} ?$ & $\mathrm{E}$ & $\mathrm{S}$ & \\
\hline A1656 & $\mathrm{NCF}$ & & $\mathrm{U}$ & $\mathrm{E}$ & & $\mathrm{S}$ & A 3562 & $\mathrm{CF}$ & $\mathrm{S}$ & & $\overline{\mathrm{D}}$ & $\mathrm{S}$ & \\
\hline A1750 & $\mathrm{NCF}$ & $\mathrm{U}$ & & $\mathrm{C}$ & $\mathrm{S}$ & $\mathrm{S}$ & A3571 & $\mathrm{CF}$ & $\mathrm{U}$ & $\mathrm{S}$ & $\mathrm{U}$ & & \\
\hline A1795 & $\mathrm{CF}$ & $\mathrm{U}$ & $\mathrm{S}, \mathrm{U}$ & $\mathrm{U}$ & $\mathrm{U}$ & & A 3627 & $\mathrm{NCF}$ & & & & & $\mathrm{s}$ \\
\hline A2029 & $\mathrm{CF}$ & $\mathrm{U}$ & U & $\mathrm{U}$ & $\mathrm{U}$ & & 2A $0335+096$ & $\mathrm{CF}$ & & & $\mathrm{U}$ & $\mathrm{U}$ & \\
\hline
\end{tabular}

structures denoted by Jones \& Forman (1999) as S in Table 1 we denoted as $\mathrm{U}$.

In the Jones and Forman catalogue (1999) clusters classified as $\mathrm{D}+\mathrm{P}+\mathrm{C}$, this is clearly substructured ones constitute $22 \%$ of the sample, while $\mathrm{D}+\mathrm{P}+\mathrm{C}+\mathrm{E}$ clusters formed $36 \%$ of the sample. Elongated clusters (E), when spherical symmetry is disturbed, are very good candidates for clusters with substructures. Among clusters from the Table 1 subsamples $\mathrm{D}+\mathrm{P}+\mathrm{C}$ constitute $25 \%$ and $22 \%$ of the total sample for $\mathrm{CF}$ and NCF clusters respectively. The single objects, not exhibit subclustering are only among $\mathrm{CF}$ clusters. The $\mathrm{D}+\mathrm{P}+\mathrm{C}+\mathrm{E}$ types constitute $50 \%$ of the CF sample and $89 \%$ of NCF sample respectively.

Due to rather small number it is not easy to perform meaningful statistical studies of the frequency of occurence of substructures among CF and NCF objects. However this frequency is higher among NCF than CF clusters. This finding is in agreement with Schuecker et al. (2001) conclusion.

From our considerations follow also that substructures are observed also among $\mathrm{CF}$ clusters. We can note that in the sample there are $6 \mathrm{cD}$ having cooling flow and 3 objects without this phenomenon. All NCF cD clusters have substructures, while among CF cD clusters only half. Oegerle \& Hill (2001) noted that $\mathrm{cD}$ clusters dynamically are similar to other clusters with similar richness. The possible difference in substructure occurence among $\mathrm{CF}$ and NCF $\mathrm{cD}$ clusters points toward the strong enviromental effects among these clusters.

\section{References}

Biviano, A., Katgert, P., Thomas, T., Adami, C., 2002, A\&A 387, 8

Dr Grandi, S., Molendi, S., 2001, ApJ 551, 153

De Grandi, S., Molendi, S., 2002, ApJ 567, 163

Escalera, E., Biviano, A., Girardi, M., Mardirossian, F., Mazure, A., Mezzetti, M., 1994, ApJ 423, 539

Jones, C., Forman, W., 1999, ApJ 511, 65

Oegerle, W.R., Hill, J.M., 2001, AJ 122, 2858

Plionis, M., 2001, astro-ph/0110616

Plionis, M., 2002, ApJL 572, 67

Schuecker, P., Boehringer, H., , Reiprich, T.H., Foretti, L., 2001, A\&A 376, 408 


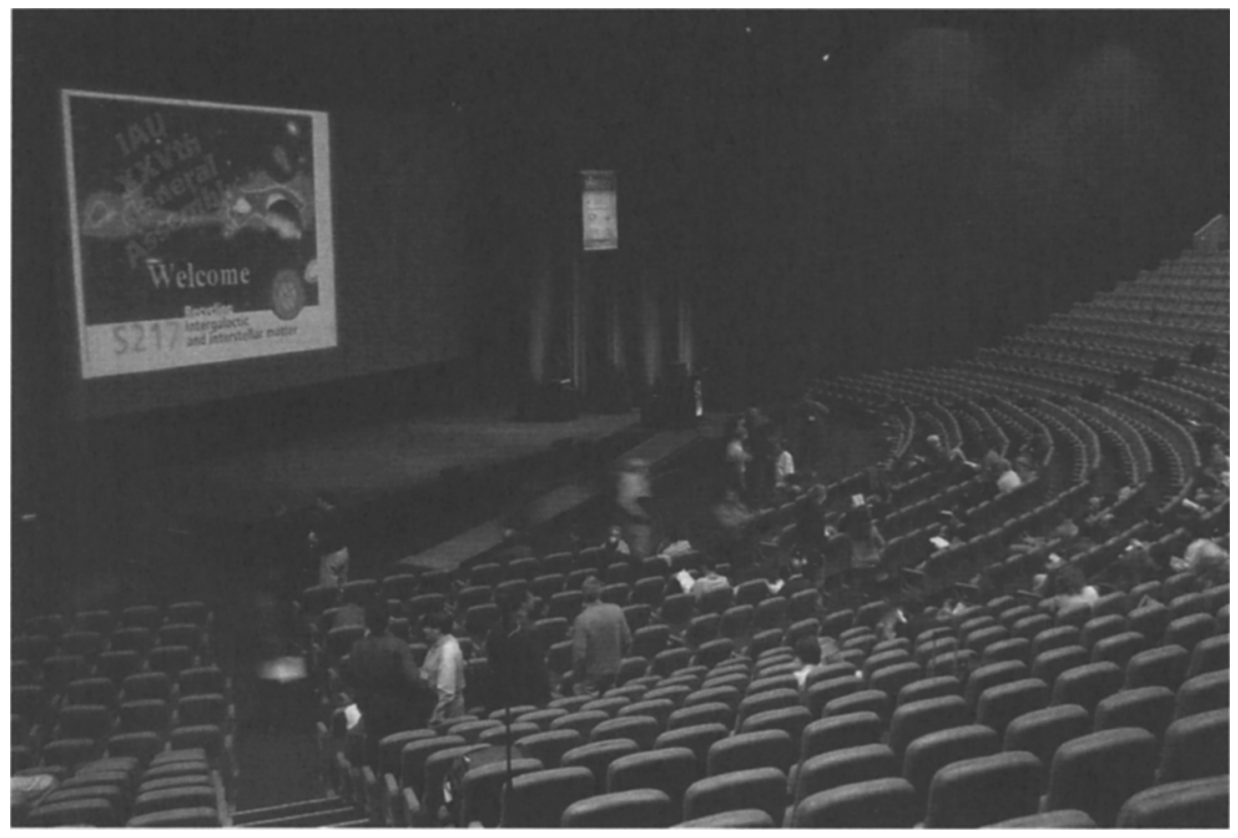

View of Harbourside Auditorium 1 hosting IAU symposium 217, inside the Convention Centre at Darling Harbour. The 2550-seat room slowly fills up ... (courtesy of Ángel Rafael López-Sánchez) 